\title{
Towards a systematic nationwide screening strategy for MODY
}

\author{
Beverley Shields $^{1} \cdot$ Kevin Colclough ${ }^{2}$
}

Received: 5 January 2017 / Accepted: 11 January 2017 /Published online: 29 January 2017

(C) Springer-Verlag Berlin Heidelberg 2017

\begin{abstract}
MODY is an early-onset monogenic form of diabetes. Correctly identifying MODY is of considerable importance as diagnosing the specific genetic subtype can inform the optimal treatment, with many patients being able to discontinue unnecessary insulin treatment. Diagnostic molecular genetic testing to confirm MODY is expensive, so screening strategies are required to identify the most appropriate patients for testing. In this issue of Diabetologia, Johansson and colleagues (DOI 10.1007/s00125-016-4167-1) describe a nationwide systematic screening approach to identify individuals with MODY in the paediatric age range. They focused testing on patients negative for both GAD and islet antigen 2 (IA-2) islet autoantibodies, thereby ruling out those with markers of type 1 diabetes, the most common form of diabetes in this age group. This commentary discusses the advantages and limitations of the approach, and the caution required when interpreting variants of uncertain pathogenicity identified from testing whole populations rather than targeting only patients with a strong MODY phenotype.
\end{abstract}

Keywords Islet autoantibodies $\cdot$ Maturity onset diabetes of the young $\cdot$ MODY $\cdot$ Monogenic diabetes $\cdot$ Variants

Beverley Shields

B.Shields@exeter.ac.uk

1 University of Exeter Medical School, University of Exeter, RILD Building - Level 3, Barrack Road, Exeter EX2 5DW, UK

2 Department of Molecular Genetics, Royal Devon and Exeter NHS Foundation Trust, Exeter, UK

\author{
Abbreviations \\ IA-2 Islet antigen 2 \\ NCDR Norwegian Childhood Diabetes Registry
}

\section{MODY: a non-insulin dependent genetic subtype of diabetes}

MODY is a clinically heterogeneous form of young-onset diabetes caused by a mutation in a single gene. Diagnosing the specific genetic cause is crucial for the patient as it can inform the optimal treatment and management of their diabetes leading to improved glycaemic control and quality of life [1]. Individuals with the most common causes of MODY have been shown to be extremely sensitive to sulfonylureas (HNF1A or HNF4A MODY) [2, 3] or require no pharmacological treatment (GCK MODY) [4]. Patients can only benefit from targeted treatment if they are correctly diagnosed, but identifying these individuals can be challenging.

\section{Recognising MODY is difficult}

Despite the importance of a correct diagnosis of MODY, it is still misdiagnosed as type 1 or young-onset type 2 diabetes in the majority of cases [5]. The high rates of misclassification are likely to be a consequence of both the unfamiliarity of MODY amongst clinicians and the difficulty of discriminating it from the more common forms of diabetes. MODY was first described by R.B. Tattersall in 1974 [6], and was characterised by non-insulin dependence, young age of onset (usually before the age of 25) and an autosomal dominant pattern of inheritance. These traditional clinical criteria, although strongly predictive of MODY, have been found to miss around 
half of all MODY cases [5]. The family history of diabetes can often be missed or unknown, and the young age of onset often leads to misdiagnosis as type 1 diabetes, so that patients are inappropriately treated with insulin and the non-insulin dependence goes unrecognised.

\section{Screening approaches are needed as diagnostic molecular genetic testing is expensive}

The expense of diagnostic molecular genetic testing has prohibited universal testing for MODY, and it is clear that traditional clinical criteria alone are insufficient for determining who to test. Therefore, screening approaches are essential to gain estimates of the true prevalence and to identify misdiagnosed MODY patients to ensure they benefit from optimal treatment. To date, several studies have screened whole paediatric clinic populations and have estimated MODY to represent between $1.1 \%$ and $4.2 \%$ of all children with diabetes [7-10]. The SEARCH for Diabetes in Youth study has been the largest to date [9], using a systematic approach, sequencing genomic DNA from 586 children who were negative for islet autoantibodies and had a fasting C-peptide $>0.8 \mathrm{ng} / \mathrm{ml}$. In this study, mutations for the three main MODY genes (HNF1A, HNF4A or GCK) were identified in $8 \%$ of the cohort, suggesting an overall population prevalence of $1.2 \%$. However, this and other studies employing systematic screening strategies have been limited to a few centres [9-11].

\section{A nationwide screening strategy for MODY}

In this edition of Diabetologia, Johansson and colleagues describe the first nationwide systematic screening programme for MODY [12]. The Norwegian Childhood Diabetes Registry (NCDR) captures over $96 \%$ of children diagnosed with diabetes making it an ideal population for a national study. All children diagnosed with diabetes in Norway are routinely tested for GAD and islet antigen 2 (IA-2) islet autoantibodies and, as these are highly sensitive and specific markers for type 1 diabetes, all patients negative for both islet autoantibodies were selected for diagnostic molecular genetic testing for MODY. Choosing to focus only on those who are antibody-negative poses an appropriate screening strategy to enrich for potential MODY. By ruling out those with markers of type 1 diabetes, the most common form of diabetes in this age group, they leave a much lower number of more appropriate individuals on which to perform genetic testing. Furthermore, positive islet autoantibody levels are rare in MODY patients, with rates similar to that seen in the non-diabetic population [13], so the number of cases missed by this approach should be minimal.
All antibody-negative diabetes patients and the same number of age- and sex-matched antibody-positive controls were tested for mutations in 13 genes associated with MODY using targeted next generation sequencing, with the main focus being placed on the five most common MODY genes in Norway: HNF1A, HNF4A, HNF1B, GCK and INS. Variants identified were confirmed by Sanger sequencing and classified using a five-tier scoring system, commonly used in clinical diagnostics laboratories, to determine the likely pathogenicity.

Of the whole national register of children with diabetes, $469(12 \%)$ were antibody-negative, resulting in a substantial reduction in the number of children tested for MODY compared with universal testing. Of these children, 6.5\% carried variants defined as class $3-5$ in the five main MODY genes, with 19 (4.1\% of antibody-negative children) carrying variants defined as class 4 or 5 (likely pathogenic or pathogenic) i.e. those who would be reported as MODY in clinical practice. In the antibody-positive cohort, $2.4 \%$ were identified as having variants in class $3-5$, but all except one of the reported variants were class 3 and so of uncertain pathogenicity. The one child carrying the class 4 variant in the antibody-positive group had an $\mathrm{HbA}_{1 \mathrm{c}}$ of $13.1 \%$ $(120 \mathrm{mmol} / \mathrm{mol})$, consistent with a dual diagnosis of type 1 diabetes and GCK MODY.

\section{Diagnosing MODY based on population screening: clinical review is still important}

Testing all those who are autoantibody-negative is a far simpler screening strategy compared with the current approach used for clinical referrals, which requires a review of each individual's case. Furthermore, it is an approach that can easily be rolled out nationally for determining who to test for MODY. However, the paper highlights the potential problems introduced by population screening. As seen in this study, a large proportion of variants of uncertain pathogenicity may be identified, requiring additional scrutiny following diagnostic testing. This is exacerbated further when considering screening of very rare, putative genetic causes of monogenic diabetes, offered as part of targeted capture, where there is little additional evidence in the literature to support or refute the association of these genes and their variants with MODY.

Determining which variants are pathogenic can be difficult. Rare variants in MODY genes previously reported as causal have been identified in normoglycaemic individuals and are therefore likely to be benign variants that do not cause monogenic diabetes [14]. This highlights the limitations of disease variant databases and the need for caution in interpreting published MODY-causing variants. Rare functional variants in known MODY genes are enriched in 
individuals with type 2 diabetes and are likely to be late-onset type 2 predisposition variants with a modest effect size [15]. Furthermore, MODY mutations previously thought to be highly penetrant have been found to have reduced or incomplete penetrance, and therefore can be identified more frequently in age-matched normoglycaemic individuals compared with other highly penetrant mutations [16]. Variation in MODY genes can thus be seen as a spectrum, moving from highly penetrant pathogenic mutations causing MODY, to pathogenic MODY-causing mutations with markedly reduced penetrance, through to variants that do not cause MODY but are late-onset type 2 diabetes risk alleles with modest effect size.

The likelihood of a variant of uncertain pathogenicity representing a genuine MODY mutation needs to be considered in light of the prior probability for MODY. In clinical practice, where patients are referred on the basis of conventional clinical criteria for MODY, the probability of MODY prior to genetic testing is high, with over half of those referred fitting traditional criteria (not insulin treated, diagnosed $<25$ years and a parent affected) having a confirmed diagnosis of MODY [5]. With a high prior probability, 'positive' genetic test results will lead to a higher positive predictive value (post-test probability) for MODY.

Such strict criteria, however, do miss a large proportion of people with MODY, which is why it is important to consider broader screening approaches. But in this setting more caution is required in interpreting variants, as the prior probability of having MODY based solely on being negative for both GAD and IA-2 antibodies is much lower than that based on clear clinical criteria (only $6.5 \%$ of antibody-negative children were identified as having variants in the five main MODY genes). So a positive genetic test result in this group will be associated with a lower positive predictive value than the same result carried out in those with a strong MODY phenotype. This means, in the case of variants of uncertain pathogenicity, they are more likely to represent genuine MODY mutations when the prior probability for MODY is higher.

Additional criteria are therefore critical for interpreting variants of uncertain pathogenicity. This is highlighted most clearly when examining the variants reported in the antibody-positive individuals: $2.4 \%$ of the antibody-positive cohort were carrying variants of class 3 or 4 , but in all these cases clinical features were inconsistent with MODY, with all (where reported) being insulin treated, many having very low C-peptide and those with $G C K$ variants having $\mathrm{HbA}_{1 \mathrm{c}}$ much higher than the expected range [17], suggesting MODY was unlikely in these cases. Therefore, none of the results in the antibody-positive cohort would result in a change in the management of the patient's diabetes. This is a reassuring finding for their proposed population screening approach, suggesting very few cases are likely to be missed, and is consistent with what would be expected given previous case-control studies [13], demonstrating the limited use of diagnostic molecular genetic testing for MODY in antibody-positive patients. For the 11 antibody-negative patients who had class 3 variants of unknown pathogenicity, only three of these variants would have been reported by the UK clinical diagnostic testing laboratory, and additional information would have been required from both the affected individual and family members to fully determine pathogenicity.

\section{Can patients with a positive genetic test result stop insulin treatment? The outcome that really matters}

In all these cases, the important test would be whether patients can successfully stop their insulin treatment. Unfortunately, this information was missing from the paper, but is the crucial reason for testing diabetes patients for MODY. The major impact of finding a MODY mutation is that treatment can be tailored to the specific genetic cause. In the case of children, where the majority of cases are misdiagnosed as having type 1 diabetes, correctly diagnosing MODY early in the course of diabetes should prevent many years of unnecessary insulin treatment. Therefore, it would be vital to follow these individuals up to ensure the best possible outcomes following the genetic diagnosis.

\section{Lower prevalence than seen in previous populations: screening in young adult age groups needs to be considered}

Although nationwide screening has been conducted, of the $12 \%$ of patients who were antibody-negative, only $4.1 \%$ had likely pathogenic or pathogenic variants (class 4 or 5), leading to a prevalence of $0.5 \%$ of children with diabetes, which is at the lower end of previous estimates described in this age group. There are many possible reasons for this that the authors themselves acknowledge. The NCDR only captures children diagnosed up to the age of 15 , whereas other studies have screened slightly older populations up to the age of 20 . With the peak age at diagnosis of people with MODY being around 15-20 years of age (derived from UK referrals data [5]), a younger age cut-off may miss many of those cases. Additionally, very few cases of GCK MODY were identified, but the authors propose that this is most likely due to the NCDR only containing patients admitted to hospital with diabetes, and that there are likely to be many more in outpatient settings that have not been detected. Screening for MODY patients outside the hospital setting and in older age groups needs to be considered. 


\section{Conclusion}

Overall, Johansson and colleagues describe a simple screening strategy that could easily be implemented nationally in clinical practice. It could be applied in the paediatric clinic but also amongst older children and young adults with diabetes. The approach is highly sensitive and substantially reduces the proportion of individuals requiring genetic testing. Caution is required when interpreting variants of unknown significance and additional clinical information should be considered alongside the genetic test results. Such screening approaches, however, are important steps forward in ensuring all MODY patients are correctly diagnosed so they can receive the optimal treatment and their family members are followed up appropriately.

Acknowledgements We would like to thank A. Hattersley, M. Shepherd and A. Jones, University of Exeter Medical School, for their helpful comments on the draft of the manuscript.

Funding BS is a core member of the National Institute of Health Research (NIHR) Exeter Clinical Research Facility.

Duality of interest The authors declare that there is no duality of interest associated with this manuscript.

Contribution statement Both authors were responsible for drafting the article and revising it critically for important intellectual content. Both authors approved the version to be published.

\section{References}

1. Rubio-Cabezas O, Hattersley AT, Njolstad PR et al (2014) ISPAD clinical practice consensus guidelines 2014. The diagnosis and management of monogenic diabetes in children and adolescents. Pediatr Diabetes 15(Suppl 20):47-64

2. Pearson ER, Starkey BJ, Powell RJ, Gribble FM, Clark PM, Hattersley AT (2003) Genetic cause of hyperglycaemia and response to treatment in diabetes. Lancet 362:1275-1281

3. Shepherd M, Shields B, Ellard S, Rubio-Cabezas O, Hattersley AT (2009) A genetic diagnosis of HNF1A diabetes alters treatment and improves glycaemic control in the majority of insulin-treated patients. Diabet Med 26:437-441

4. Stride A, Shields B, Gill-Carey O et al (2014) Cross-sectional and longitudinal studies suggest pharmacological treatment used in patients with glucokinase mutations does not alter glycaemia. Diabetologia 57:54-56

5. Shields BM, Hicks S, Shepherd MH, Colclough K, Hattersley AT, Ellard S (2010) Maturity-onset diabetes of the young (MODY): how many cases are we missing? Diabetologia 53:2504-2508

6. Tattersall RB (1974) Mild familial diabetes with dominant inheritance. Q J Med 43:339-357

7. Fendler W, Borowiec M, Baranowska-Jazwiecka A et al (2012) Prevalence of monogenic diabetes amongst Polish children after a nationwide genetic screening campaign. Diabetologia 55:26312635

8. Irgens HU, Molnes J, Johansson BB et al (2013) Prevalence of monogenic diabetes in the population-based Norwegian Childhood Diabetes Registry. Diabetologia 56:1512-1519

9. Pihoker C, Gilliam LK, Ellard S et al (2013) Prevalence, characteristics and clinical diagnosis of maturity onset diabetes of the young due to mutations in HNF1A, HNF4A, and glucokinase: results from the SEARCH for Diabetes in Youth. J Clin Endocrinol Metab 98: 4055-4062

10. Shepherd M, Shields B, Hammersley S et al (2016) Systematic population screening, using biomarkers and genetic testing, identifies $2.5 \%$ of the U.K. pediatric diabetes population with monogenic diabetes. Diabetes Care 39:1879-1888

11. Chambers C, Fouts A, Dong F et al (2015) Characteristics of maturity onset diabetes of the young in a large diabetes center. Pediatr Diabetes 17:360-367

12. Johansson BB, Irgens HU, Molnes J et al (2016) Targeted nextgeneration sequencing reveals MODY in up to $6.5 \%$ of antibodynegative diabetes cases listed in the Norwegian Childhood Diabetes Registry. Diabetologia. doi:10.1007/s00125-016-4167-1

13. McDonald TJ, Colclough K, Brown R et al (2011) Islet autoantibodies can discriminate maturity-onset diabetes of the young (MODY) from type 1 diabetes. Diabet Med 28:1028-1033

14. Flannick J, Beer NL, Bick AG et al (2013) Assessing the phenotypic effects in the general population of rare variants in genes for a dominant Mendelian form of diabetes. Nat Genet 45:1380-1385

15. Fuchsberger C, Flannick J, Teslovich TM et al (2016) The genetic architecture of type 2 diabetes. Nature 536:41-47

16. Laver TW, Colclough K, Shepherd M et al (2016) The common p.R114W HNF4A mutation causes a distinct clinical subtype of monogenic diabetes. Diabetes 65:3212-3217

17. Steele AM, Wensley KJ, Ellard S et al (2013) Use of $\mathrm{HbA}_{1 \mathrm{c}}$ in the identification of patients with hyperglycaemia caused by a glucokinase mutation: observational case control studies. PLoS One 8: e65326 